\section{Change detection and off-frequency listening: A reply to Leshowitz and Wightman*}

\author{
NEIL A. MACMILLAN \\ Brooklyn College of the City University of New York \\ Brooklyn, New York 11210
}

Leshowitz and Wightman's (1972) account of the fact that recognition of increments and decrements in intensity improves with signal duration relative to detection of increments and decrements is found wanting. Not only does their interpretation fail to explain the occurrence of the effect in several different stimulus situations, but it is inconsistent with the results of detection experiments in which either signal duration or the signal polarity is uncertain.

Macmillan (1971) reports two types of evidence for change detection, a mode of detection in which signals are not identified, and in which sensitivity is not dependent on duration. First, recognition of increments and decrements in a pure-tone background is shown to improve, relative to detection of the same signals, as duration increases. (This effect will be referred to as the detection-recognition disparity.) Second, when the $O$ does not know whether the signal to be detected will be an increment or a decrement, his performance is inferior to detection of known signals only at long durations. Macmillan (1971) hypothesized that the change detector combines with an integrative detector in detection experiments; in recognition experiments, the change detector is not used, or at any rate, does not help.

An alternative explanation of these results, proposed by Leshowitz and Wightman (1972) is based on their (1971) variable filter model. They note that the energy spectrum of a short pure-tone burst has a greater proportion of its energy off frequency than does a longer burst, and they suggest that the $O$ moves an internal filter away from the signal frequency to take advantage of this unmasked "energy splatter." This strategy is helpful in detecting short-duration signals, but it is not useful in recognition; thus, the detection-recognition disparity is accounted for.

Two aspects of this explanation are noteworthy. First, the model predicts that the effect of duration on threshold intensity will be slight, which is in fact what Macmillan (1971) found. This prediction arises from the fact that the absolute amount of energy off frequency does not change as signal duration is varied; if only off-frequency energy is relevant

*Preparation of this note was aided by a grant from the City University of New $Y$ ork Faculty Research Award Program. to the O's decision, no effect of duration on intensity will be found at all. Thus, the detection-recognition disparity is tied, in this explanation, to a shallow intensity-duration relation.

Second, the model postulates that the $O$ has control over the listening process, since he must "maximize the ratio of signal energy to masker energy at the output of the auditory filter [Leshowitz \& Wightman, 1972]." Presumably moderate imprecision in the setting of the filter would not drastically change the predictions of the model, but it is clear that the predictions are dependent on the ability of the $\mathrm{O}$ to locate his filter off frequency in detection experiments, on frequency in recognition. Similarly, as will be shown, the predictions made by this model for uncertain detection experiments depend on whether the $O$ in detection sets the filter differently for different durations.

Both of these critical points in Leshowitz and Wightman's explanation are suspect; I shall discuss each of them briefly.

\section{TEMPORAL INTEGRATION}

The assertion that the detection-recognition disparity occurs only when the intensity-duration relation is shallow has recently been refuted by Macmillan (1972). In that paper, the disparity is shown to occur in many stimulus situations, some of which are characterized by a large degree of temporal integration, some by smaller amounts. For example, the addition of a noise floor increases the amount of temporal integration, but it does not affect the detection-recognition disparity (contrary to Leshowitz and Wightman's explicit prediction) or the duration effect on signal uncertainty. The detection-recognition disparity also occurs when the signals are increments and decrements in noise, although the intensity-duration function is not unusual.

The implications of these findings seem to be: (1) the detection-recognition disparity occurs whether or not the $O$ is listening off frequency, and (2) off-frequency listening may in fact occur in situations where it is profitable, as demonstrated by the shallow intensity-duration relations in tone experiments.

\section{OBSERVER CONTROL}

The notion of an internal filter is pervasive in psychoacoustics, and how much control the $O$ has over the filter is an important, largely unresolved question (see Penner, 1972, and references therein). Leshowitz and Wightman (1972) suggest that not only does the $O$ locate his filter differently in detection than in recognition, he also locates it differently for detecting signals of different durations: "It is reasonable to suppose that as signal duration increases the $\mathbf{O}$ moves his filter toward the signal frequency." In fact, this assumption is necessary if Leshowitz and Wightman are to account for the uncertain detection results of Macmillan (1971, Experiment 4): Os' performance in detection is poorer if they are uncertain whether the signal will be an increment or a decrement than if they are not uncertain, and this decline is greatest at the longest duration. A straightforward signal detection theory analysis predicts a constant decline. Macmillan accounts for the duration effect by invoking change detection, which is unaffected by this type of uncertainty, and which, therefore, reduces the decline in performance at short durations, where that mechanism is most effective. Leshowitz and Wightman can only explain the result as being due to a shift towards on-frequency listening at the longest duration. Thus, if the off-frequency listening hypothesis is to account for Macmillan's results, the location of the internal filter in detection experiments must be a function of signal duration.

Direct evidence on whether the filter does shift in this way is contained in Experiment 5 of Macmillan's (1971) paper. Detection of increments in a pure tone was the same whether the duration of the increments $w$ as constant within blocks or randomized. Leshowitz and Wightman's model is consistent with this result only if the location of the internal filter is assumed not to change with duration in the blocks condition. Thus, their model can account for the results of either of these two experiments (uncertain detection or randomized duration detection), but not both. (The model can be salvaged, as far as these two experiments are concerned, by supposing that the filter is kept fixed with duration when all signals are increments, but is changed when signals may be increments or 
decrements. This seems ad hoc.)

In view of these two separate points of disagreement between Leshowitz and Wightman's model and the data, it seems fair to reject the model as an explanation for these data. More important, however, are the severe restrictions which these data place on any model for auditory detection. The observation that randomizing signal duration does not affect performance is problematic not only for Leshowitz and Wightman, but for virtually all models of detection, since such models consistently assume that the $O$ has at least approximate knowledge of the signal interval. If this were true, detection performance would be better when the signal was known than when it was unknown; since there is no difference between the two conditions, the $O$ must not have (or anyhow, use) knowledge of the signal duration.

Thus, models which postulate the accumulation of information for the period of the observation interval require modification. Models are needed in which the stimulus is viewed as being processed over time in such a manner as to make exact knowledge of the signal duration unnecessary. Few such models exist, although Luce and Green (1972) have proposed a "neural timing theory" from which models having this property (see Macmillan, 1972) may be developed.

\section{REFERENCES}

LESHOWITZ, B. \& WIGHTMAN, F. L On-frequency masking with continuous sinusoids. Journal of the Acoustical Society of America, 1971, 49,
1180-1190.

LESHOWIT, B. \& WIGHTMAN, F. L. On the importance of considering the signal's frequency spectrum: Some comments on Macmillan's "Detection and recognition of increments and decrements in auditory intensity" experiment. Perception \& Psychophysics, 1972, 12, 209-210.

LUCE. R. D. \& GREEN, D. M. A neural timing theory for response times and the psychophysics of intensity. Psychological Review, 1972, 79, 14-57.

MACMILLAN, N. A. Detection and recognition of increments and decrements in auditory intensity. Perception \& Psychophysics, 1971, 10, 233-238.

MACMILLAN, $N$, Detection and recognition of intensity changes in tone and noise: The detection-recognition disparity. Perception \& Psychophysics. 1971,10 (4A), 233-239.

PENNER, M. J. The effect of payoffs and cue tones on detection of sinusoids of uncertain frequency. Perception \& Psychophysics, 1972, 11, 198-202.

(Accepted for publication May 2, 1972.) 